\title{
Beneficios del ejercicio en la insuficiencia cardíaca
}

\author{
Jorge Márquez ${ }^{1}$, Gustavo Suárez ${ }^{1}$, Jhony Márquez ${ }^{2}$ \\ 1 Universidad de Antioquia, Medellín, Colombia \\ 2 Clínica Las Américas, Medellín, Colombia
}

La insuficiencia cardíaca es un síndrome incapacitante y frecuente que genera gran morbimortalidad y sus principales síntomas son fatiga, disnea e intolerancia al ejercicio. El ejercicio aeróbico, el de fuerza o el entrenamiento combinado tienen beneficios sobre la patogénesis, síntomas específicos, tolerancia al ejercicio, capacidad física, fuerza muscular, pronóstico, calidad de vida y aspectos psicosociales de los pacientes. El entrenamiento regular se asocia con múltiples adaptaciones centrales y periféricas con mecanismos claros que explican la mejoría del paciente con insuficiencia cardíaca; entre ellos están el cambio de la función cardíaca, respiratoria, vascular, neurohumoral, muscular, inflamatoria, antioxidante, etc. Revisiones sistemáticas, meta-análisis y estudios con buen diseño metodológico han encontrado disminución de las admisiones hospitalarias, tiempo de hospitalización, disminución de los costos, mejoría de los síntomas y efectos adversos y reducción de la mortalidad. El ejercicio adecuadamente prescrito es seguro y eficaz en el paciente con insuficiencia cardíaca. Para optimizar los beneficios del ejercicio regular se debe garantizar una buena adherencia al plan de entrenamiento.

Palabras claves: Ejercicio, ejercicio aeróbico, entrenamiento de resistencia, insuficiencia cardíaca. 


\section{Beneficial effects of exercise in patients with heart failure}

Heart failure is a common and disabling syndrome that generates high morbidity and mortality and its main symptoms are fatigue, dyspnea and exercise intolerance. Aerobic exercise, strength training or combined exercise have benefits on the pathogenesis, specific symptoms, exercise tolerance, physical capacity, muscle strength, prognosis, quality of life and psychosocial aspects of patients. Regular training is associated with multiple central and peripheral adaptations explained with clear mechanisms explaining the improvement of patients with heart failure. Included are the changes in cardiac, respiratory, vascular,

\section{Introducción}

La Insuficiencia Cardíaca (IC) es un síndrome incapacitante y la mayoría de las veces es secundario a enfermedad coronaria o hipertensión arterial ${ }^{1,2}$. Es una de las enfermedades cardiovasculares más frecuentes y genera alta morbi-mortalidad1-3. Su prevalencia varía, según la edad, de 1-2\% en personas de 55-64 años a $\geq 10 \%$ en los mayores de 70 años1. Consume aproximadamente el 2\% del presupuesto del Servicio Nacional de Salud en el Reino Unido y el costo anual en USA es US\$ 372.000 millones y se estima que 5.700 .000 personas la tienen actualmente. 2,4

Además, se asocia con comorbilidades no cardíacas que afectan su desarrollo, progresión y respuesta al tratamiento, tales como disfunción pulmonar, trastornos respiratorios del sueño, disfunción renal y hepática, anemia, diabetes, trastornos tiroideos, miopatía, depresión y compromiso cognitivo. 5

Los pacientes con IC presentan varios síntomas pero los más comunes son la disnea, la fatiga y el compromiso progresivo de la tolerancia al ejercicio que afectan la calidad de vida y las actividades de la vida diaria ${ }^{\mathbf{1 , 6}}$. Los síntomas y la capacidad de ejercicio se usan para clasificar la severidad de la enfermedad y para evaluar la respuesta al tratamiento 6,7 . Para ello se utiliza generalmente la clasificación de la NYHA, que tiene una variabilidad interobservador considerable y es insensible a cambios importantes en la capacidad de ejercicio. Las correlaciones son pobres entre los síntomas y el grado de compromiso cardíaco en reposo, y entre los síntomas y el pronóstico en IC. 1,7

Antes del final de la década de los 80 , el reposo en cama y la restricción de la actividad física se recomendaba neurohormonal and muscular functions, anti-inflammatory and anti-oxidant effects. Systematic reviews, meta-analysis and studies with good methodological design found decreased hospital admissions, length of stay, lower costs, improved symptoms and adverse effects and mortality reduction. Properly prescribed exercise is safe and effective in patients with heart failure. Good adhesion to the training plan is required to maximize the benefits of regular exercise in patients with heart failure.

Keywords: Exercise, aerobic exercise, resistance training, heart failure.

para todos los estadios de IC. Esto puede llevar a atrofia muscular, embolismo pulmonar, trombosis venosa, reducción de la tolerancia al ejercicio y exacerbación de los síntomas ${ }^{8}$. En las últimas décadas, se han realizado múltiples investigaciones que evidencian los beneficios y seguridad del ejercicio en el paciente con IC ${ }^{\mathbf{2}, 7,9}$, tanto que las guías NICE, ACCF/AHA y ESC enfatizan la importancia de las intervenciones en el estilo de vida, como el ejercicio regular en el manejo de la IC para mejorar la capacidad funcional y los síntomas (Recomendación Ia, Nivel de evidencia A). $\mathbf{1 , 6 , 1 0}$

\section{Tolerancia al ejercicio en IC}

La IC, tanto con FE preservada como reducida, se caracteriza por deterioro progresivo de la capacidad funcional o de la tolerancia al ejercicio y disminución del consumo máximo de oxígeno (VO2máx) secundario a varios mecanismos, que incluyen principalmente, disfunción cardíaca, anormalidades en el flujo sanguíneo periférico, disfunción endotelial, inflamación, alteraciones del músculo esquelético, defectos ventilatorios, disfunción neurohormonal y factores psicosociales $2,11,12$

La capacidad para hacer ejercicio aeróbico - determinada por el VO2máx, depende del gasto cardíaco (GC) y de la diferencia arteriovenosa de Oxígeno (DavO2). En la persona normal el GC y la DavO2 aumentan y la resistencia vascular periférica (RVP) disminuye durante el ejercicio aeróbico. En la IC no aumenta adecuadamente el GC ( $\leq 50 \%$ GC Máximo) debido al mínimo incremento del volumen de eyección y a una menor frecuencia cardíaca máxima lograda en una carga menor de ejercicio ${ }^{\mathbf{1 3}}$. La falla para incrementar el volumen de eyección deriva principalmente de la alteración de la contractilidad, 
respuesta beta adrenérgica reducida, RVP aumentada mayor actividad del sistema simpático y del eje reninaangiotensina - e inadecuada respuesta vasodilatadora al ejercicio y, eventualmente, regurgitación mitral ${ }^{2,13}$. Es importante conocer que existe poca correlación entre la capacidad funcional, o el rendimiento en el ejercicio y la fracción de eyección, u otros índices hemodinámicos en reposo ${ }^{14}$, pero, sí se correlacina con los parámetros de disfunción diastólica en pacientes normales y con IC. Sin embargo, el estado de salud promedio y el pronóstico se predicen mejor con índices de tolerancia al ejercicio determinados en una prueba de esfuerzo cardiopulmonar ${ }^{15}$ que también debe considerarse para detectar isquemia miocárdica reversible, en pacientes candidatos a trasplante cardíaco o soporte circulatorio mecánico y para ayudar en la prescripción del ejercicio. ${ }^{1}$

Las anormalidades periféricas contribuyen al deterioro funcional y para muchos autores, parecen ser las responsables directas de la sintomatología de la IC $\mathbf{2 , 9}$, 13. La disfunción ventricular disminuye el flujo periférico y activa un estado catabólico causando miopatía que acentúa la disnea y la fatiga y, además, estimula los ergo-receptores musculares, llevando a hiperventilación y excitación simpática e inhibición vagal que produce vasoconstricción e incremento de la poscarga que causa más deterioro de la función cardíaca, formando un círculo vicioso. Lo anterior resulta en empeoramiento de la tolerancia al ejercicio, miopatía (cambios en la morfología, función y metabolismo del músculo con atrofia muscular), disminución de la vascularización, cambio de la fibra muscular a un fenotipo más rápido (atrofia de fibras tipo IIa y $\operatorname{IIb}(\mathrm{x})$ con disminución de IIa) y disminución de la resistencia a la fatiga - y pudiera llevar a efectos adversos progresivos en la remodelación del ventrículo izquierdo ${ }^{2}$, 13,16. También se reporta disfunción vascular y endotelial, inflamación persistente, imbalance autonómico y hormonal, disminución de la densidad capilar y capacidad oxidativa y metabólica del músculo esquelético, además de disminución de la actividad de la citrato sintasa y la creatin kinasa. ${ }^{\mathbf{9}, 11}$.

Asimismo, la sarcopenia es más acelerada en pacientes con IC y el área transversal del músculo esquelético y la fuerza son predictores independientes de tolerancia al ejercicio, pronóstico clínico y supervivencia a largo plazo, 17; el VO2Max de individuos con fuerza de piernas normal, está restringido generalmente por la reserva cardiovascular, mientras en pacientes con IC el fenotipo miopático de las piernas puede ser un factor limitante primario. $^{17}$

Otros factores que se han asociado con intolerancia al ejercicio se muestran en la Tabla 1.
Tabla 1. Otros factores asociados con intolerancia al ejercicio

Anormalidades de la función diastólica: mediciones

ecocardiográficas y el volumen indexado de la aurícula izquierda

son predictores independientes y confiables de capacidad de

ejercicio en pacientes con disfunción diastólica aislada.

La debilidad de los músculos inspiratorios contribuye a la

disminución de la capacidad de ejercicio y a la disnea durante las

actividades cotidianas, y la fuerza de éstos, tiene valor pronóstico

independiente en IC.

Niveles elevados de BNP y NT-proBNP son útiles para identificar pacientes con limitación cardiocirculatoria.

Bajos niveles de testosterona circulantes se relacionan independientemente con intolerancia al ejercicio en hombres con IC.

Resistencia a la insulina explicado en parte por disminución de reserva de flujo coronario.

Falla energética: disminución capacidad oxidativa (biogénesis mitocondrial), disminución kinasas de fosfotransferencia, disminución eficiencia en consumo energético, etc.

Disregulación en mediadores inflamatorios (TNF- $\alpha$ e IL-1 $\beta$ ) y especies reactivas de oxígeno.

Resistencia muscular funcional a la adiponectina

\section{Beneficios del ejercicio en IC}

El ejercicio juega un papel importante en cualquiera de los 4 estados de la IC, en su prevención, tratamiento no farmacológico, control, rehabilitación y pronóstico, siempre y cuando se prescriba de la manera adecuada 2 , 19,20 .

El entrenamiento físico, tanto aeróbico- continuo o intermitente-, como de fuerza, produce adaptaciones periféricas significativas e importantes cambios hemodinámicos centrales que contribuyen a detener o reversar el desarrollo de $\mathrm{IC}^{\mathbf{8}, 21}$, y tiene beneficios sobre la patogénesis, y en síntomas específicos, "fitness" aeróbico (tolerancia al ejercicio), fuerza muscular y calidad de vida de los pacientes, y puede tener impacto benéfico en los resultados clínicos (nivel de evidencia A) 19,21-25.

\section{Mecanismos por los cuales el ejercicio regular revierte la intolerancia al ejercicio en IC}

Los mecanismos que explican la mejoría de la tolerancia al ejercicio después del entrenamiento aeróbico (EA) son multifactoriales y pueden ser centrales o periféricos.

\section{Mecanismos Centrales}

Los pacientes con IC tienden a tener una adaptación cardiovascular al ejercicio y una recuperación más lentas ${ }^{\mathbf{2 6}}$. Varios estudios reportan mejoría de la función cardíaca en reposo y ejercicio ${ }^{2,27,28}$. Se encuentra aumento del gasto cardíaco máximo y en ocasiones el submáximo asociados con el aumento de frecuencia cardíaca pico (4 a 8\%), volumen de eyección pico y de la velocidad del llenado 
diastólico, la "compliance" y el "stiffness" diastólico del $\mathrm{VI}^{29}$ y la fracción de eyección máxima; también se describe reversión de la incompetencia cronotrópica, disminución de la poscarga e incremento de la estabilidad eléctrica del miocardio por aumento directo de la actividad parasimpática en reposo, disminución directa de la actividad simpática y de los niveles plasmáticos de norepinefrina en reposo y ejercicio, y posible incremento del umbral de fibrilación ventricular.,9,30

Además, el ejercicio aeróbico puede atenuar o aún revertir la remodelación del ventrículo izquierdo ${ }^{31,32}$, restaurar la sensibilidad al calcio y la contractilidad del cardiomiocito, y mejorar la función sistólica y diastólica. 8, 12, 20,31

Aunque falta más evidencia, el ejercicio regular puede mejorar el metabolismo energético miocárdico sumado a la función diastólica ventricular. ${ }^{12,33-35}$

Adicionalmente, el ejercicio regular tiene beneficios generales en el balance entre la demanda, suministro de oxígeno y trabajo miocárdico por sus efectos en el retraso de la progresión de la aterosclerosis, disminución del doble producto durante el ejercicio y disminución de la actividad simpática ${ }^{30}$.

\section{Mecanismos Periféricos}

Múltiples investigaciones encuentran incremento del flujo sanguíneo local, alrededor de $28 \%$, y en músculos esqueléticos metabólicamente activos ${ }^{2,14,30}$.

El ejercicio regular revierte parcialmente la activación neurohormonal de la IC explicado en parte por el aumento de la variabilidad de la frecuencia cardíaca, la disminución de la actividad simpática y los niveles de BNP y NT-proBNP correlacionados con los cambios en el VO2 max ${ }^{36}$. Ocurre mejoría de la función $(\uparrow$ vasodilatación 2.5 veces y flujo $(50 \%)$ dependiente del endotelio - incremento de la síntesis y liberación de óxido nítrico y expresión de la óxido nítrico sintasa endotelial (eNOS) - y aumenta la reactividad vascular ${ }^{\mathbf{1 4 , 3 0}}$; favorece angiogénesis - $\uparrow$ expresión de factor de crecimiento endotelial vascular (VEGF) y reduce el estrés oxidativo y RVP en tejidos activos. ${ }^{14,30,35}$

Con el entrenamiento se mejora la función del músculo esquelético y la utilización de oxígeno relacionada con el aumento de la fuerza y resistencia muscular, aumento de la capacidad metabólica y oxidativa, aumento del número y tamaño mitocondrial, aumentan las vías de señalización que aumentan la expresión muscular y cardíaca de PGC1a, incremento relativo del porcentaje de la cadena pesada de la miosina de las fibras tipo $\mathrm{I}^{\mathbf{1 4}} \mathbf{1 6}, \mathbf{3 5}$, y aumento de la densidad capilar. Además se reduce la apoptosis de miocitos, aumenta la expresión de IGF-1 en el músculo esquelético y cardíaco (mejora la síntesis proteica muscular a través de la activación de la vía de señalización molecular P13K/Akt) ${ }^{37,38}$, incrementa la actividad de la citrato sintasa, reduce la depleción de fosfocreatina, aumenta la expresión de isoenzimas creatin kinasa e incrementa el adenosin difosfato durante el ejercicio ${ }^{33,35}$, e incrementa la eficacia de la transferencia energética ${ }^{7,8,35}$. Además, puede reducir la expresión de la vía proteasomaubiquitina relacionada con atrofia, la cual es vital para la degradación de proteínas contráctiles y atenuar la degradación proteica inhibiendo los FOXO, relacionados con la atrofia mediada por proteólisis8; se ha encontrado normalización de la expresión de RNAm de adiponectina y AdipoR1 y reversión de alteraciones en el metabolismo de lípidos y glucosa en el músculo.

\section{Función Autonómica y Neurohormonal}

La activación del SNS y la regulación en baja del parasimpático de los pacientes con IC avanzada se relacionan con la supervivencia y contribuyen a la miopatía. El ejercicio regular reduce o revierte este imbalance documentado por disminución directa e indirecta de la actividad simpática a los tejidos periféricos en reposo y de la hiperactividad neuroendocrina, baja los niveles circulantes de catecolaminas, aumenta el tono vagal y mejora la sensibilidad de los baroreceptores y quimiorreceptores ${ }^{7}$, 39, 40. La frecuencia cardíaca de reposo y la submáxima disminuyen, mientras el doble producto en cargas submáximas aumenta más gradualmente y a niveles más bajos $^{27,38,40}$. También se mejora la respuesta cronotrópica al ejercicio: la frecuencia cardíaca es más alta en cargas máximas y más baja durante la recuperación. 38,40

Trabajos recientes muestran que 16 semanas de ejercicio regular pueden ayudar a modificar el estado neuroendocrino de reposo; se encuentran disminuciones de 26\%,32\%, $30 \%$ y $27 \%$ en angiotensina, aldosterona, vasopresina y péptido natriurético atrial respectivamente. $7,30,40$

\section{Estado Inflamatorio y Antioxidante}

Las citoquinas proinflamatorias participan en la fisiopatología de la IC. El entrenamiento físico es una estrategia terapeútica antiinflamatoria valiosa en el paciente con $\mathrm{IC}^{\mathbf{2}, \mathbf{9 , 3 7}, \mathbf{4 1}}$. El ejercicio regular disminuye las concentraciones plasmáticas y musculares de citoquinas proinflamatorias (TNF: $-37 \%$, IL-6: - 42\%, IL-1 $\beta$ : $-48 \%)^{41,42}$, aumenta la IL-10 (antiinflamatoria) y pudiera prevenir la caquexia o revertirla ${ }^{\mathbf{3 0}}$; además, el ejercicio modula el sistema inmune innato, influenciando la función de los linfocitos y macrófagos. ${ }^{37}$

El ejercicio regular tiene un potente efecto en la regulación 
en baja en la inflamación mediada por plaquetas, disminuyendo los niveles plasmáticos de ligando CD40 soluble y selectina-P y, además, modula benéficamente la respuesta inmune en la IC ya que incrementa factores antiapoptóticos y aumenta la expresión y sensibiliza la actividad del IGF-137, ${ }^{43}$, hecho asociado con disminución de apoptosis de miocitos y reversión de atrofia muscular; El ejercicio intenso disminuye marcadores periféricos de disfunción endotelial (factor estimulante de colonias granulocito-macrófago (GM-SCF), proteína 1 quimioatrayente de macrófagos (mcp-1), molécula 1 de adhesión intracelular (ICAM 1. y molécula 1 de adhesión de célula vascular (VCAM-1)). ${ }^{37}$

Por otra parte, el entrenamiento físico reduce la expresión de citoquinas proinflamatorias musculares y de la iNOS, aumenta la actividad de la Citocromo C, incrementa el "shear stress" en el endotelio vascular lo cual promueve la expresión de la eNOS, disminuye la producción de especies reactivas de oxígeno (ROS) y prostanoides e induce la expresión de la superóxido dismutasa(SOD). ${ }^{2,7,8}$

Según datos recientes, el efecto antiinflamatorio significativo del entrenamiento durante 20 semanas desaparece después de 1 año sin ejercicio sistemático documentado por el retorno a valores normales de ligando CD40 soluble y P-selectina ${ }^{44}$, lo que refuerza el concepto de la adherencia al ejercicio para optimizar los beneficios. La Tabla 2 resume algunas vías inducidas por el ejercicio en la IC.

Tabla 2 Algunas vías inducidas por el ejercicio regular en pacientes con falla cardiaca

Función Cardiaca: aumento de la sensibilidad al calcio y contractilidad miocárdica, mejoría hemodinámica, restauración de preacondicionamiento isquémico, reversión de la incompetencia cronotrópica, antiremodelación(FEVI, EDV, ESV)

Vasculatura: aumento de la eNOS, NO, SOD, función endotelial, aumento de la expresión de VEGF; disminución ROS, estrés oxidativo

Reflejos vasculares: aumento de la sensibilidad de baroreceptores arteriales y mejora el control quimioreceptor

Neurohumoral: disminución de la Angiotensina 2, receptores tipo 1 de angiotensina, ROS, actividad simpática; disminución de la Norepinefrina, vasopresina, aldosterona; disminución de $\mathrm{NO}$ y actividad vagal, HRV

Músculo: aumento de enzimas oxidativas, contenido mitocondrial, IGF-1, activación de la vía P13K/Akt, aumento de la expresión de PGC-1a; disminución del estrés oxidativo y citoquinas proinflamatorias, disminución de la miosina de fibras Ia, disminución de la expresión de la vía proteasoma-ubiquitina relacionada con atrofia, Inhibición de los los FOXO

Antinflamatorio: disminución de iNOS, TNF, IL-6, IL-1 $\beta$, CD4OL, selectina-P, GM-CSF, MCP-1, ICAM-1, VCAM-1; $\uparrow$ IL-10
Efectos del Ejercicio en la Capacidad Física y el VO2 max.

Los pacientes con NHYA II-III tienen el VO2 max entre 12 y 19 ml kg min $-^{1}$ or $40-55 \%$ debajo de los pacientes $\sin \mathrm{IC}^{8}$.

El EA prolonga la fase I de la cinética del consumo de oxígeno e incrementa la eficiencia en la pendiente de captación de oxígeno, lo cual es una medida de la función cardiopulmonar de reserva y tiene un alto valor pronóstico en la $\mathrm{IC}^{\mathbf{8}, 45}$.

El ejercicio aeróbico o combinado con entrenamiento de fuerza mejora significativamente la capacidad física y el VO2 $\max ^{25,46}$; hay una tendencia hacia la superioridad del ejercicio intenso intermitente o de intervalos vs el aeróbico continuo ${ }^{46}$ y hay evidencia de que el entrenamiento combinado maximiza los beneficios ${ }^{\mathbf{1 4}}, \mathbf{1 7}$, ${ }^{46}$. El incremento del VO2 max varía de 10 a $30 \%{ }^{\mathbf{1 3}}$ y rara vez aumenta a $40-45 \%$ 46; También mejora el VO2 en el umbral anaeróbico (hasta 25\%), el tiempo de ejercicio y la máxima carga lograda. ${ }^{\mathbf{7}} \mathbf{8}$.

Un metanálisis de entrenamiento en 2.387 pacientes encontró un incremento promedio del VO2 max de 17\% en 57 estudios que lo midieron directamente, de $17 \%$ en 40 trabajos con ejercicio aeróbico, $9 \%$ en 3 estudios de EF, $15 \%$ en 13 investigaciones con entrenamiento combinado aeróbico y de fuerza y $16 \%$ en 1 estudio con entrenamiento de los músculos inspiratorios. ${ }^{35}$

Los pacientes con falla cardíaca con fracción de eyección preservada pueden beneficiarse del entrenamiento y se ha encontrado mejoría de $30 \%$ de su capacidad funcional y VO2 max y calidad de vida. ${ }^{20}$

\section{Efectos del Ejercicio en la Función Respiratoria}

El EA causa una disminución de la ventilación minuto submáxima, menor frecuencia respiratoria y volumen corriente, menor valor de la pendiente del equivalente ventilatorio del $\mathrm{CO} 2$ y aumento del umbral anaeróbico ${ }^{8}$.

Adicionalmente, el trabajo de fuerza de miembros superiores mejora la ventilación durante el ejercicio y la resistencia de los músculos respiratorios, aliviando la disnea. El ejercicio regular reduce la sensación de disnea en reposo y ejercicio ${ }^{2}$. El entrenamiento de los músculos inspiratorios aumenta su fuerza y resistencia, y mejora la capacidad física y la calidad de vida y es una alternativa al ejercicio aeróbico en las fases iniciales en pacientes muy desacondicionados. ${ }^{8,47}$

\section{Beneficios Psicosociales}

El ejercicio regular es un enfoque terapéutico efectivo para reducir la disnea y la fatiga muscular de los pacientes con IC, promoviendo su independencia y salud 
psicosocial. Lo anterior, sumado con la mejoría de uno o más parámetros de la calidad de vida, se asocia con menos conductas de enfermedad crónica, depresión ${ }^{48}$ y mejora su sensación de bienestar. ${ }^{24,30}$

Se ha encontrado mejoría de la calidad de vida después del entrenamiento independientemente de incrementos simultáneos de la capacidad aeróbica ${ }^{7}, 21$. Un metaanálisis reciente que analizó los efectos del ejercicio regular programado en la calidad de vida relacionada con la salud de pacientes con IC usando el "Minnesota Living With Heart Failure Questionnaire" reporta una ganancia clínicamente significativa de 7,3 (3.3-11.4) puntos. ${ }^{20}$

\section{Efectos en la morbilidad y mortalidad}

Hay al menos 34 estudios y varios meta-análisis que reportan mejoría en los resultados con disminución de las admisiones hospitalarias, tiempo de hospitalización, disminución de los costos, mejoría de los síntomas y efectos adversos y reducción del riesgo relativo de muerte con ejercicio regular en IC. , $^{22}$

Dos meta-análisis similares apoyan la tendencia a la disminución de los eventos clínicos en pacientes que entrenan 23,24: El ExTraMATH encontró que 801 pacientes con seguimiento promedio 705 días pueden obtener los beneficios del ejercicio aunque los hombres mayores de 60 años, clase funcional NYHA III-IV, etiología isquémica, fracción de eyección $<27 \%$, VO2 $\max <15 \mathrm{ml} / \mathrm{Kg} / \mathrm{min}$ y duración del entrenamiento $\geq 28$ semanas obtuvieron beneficios estadísticamente significativos; además, concluye que hay evidencia de la reducción promedio en la mortalidad y en las admisiones hospitalarias. ${ }^{\mathbf{2 4}}$

Debido a que la mayoría de los estudios no han tenido poder estadístico adecuado para medir los efectos del ejercicio en los resultados clínicos se diseñó un ensayo controlado aleatorizado multicéntrico ${ }^{22}$ - HF-ACTION - para determinar la eficacia y seguridad del EA, cuyo desenlace primario compuesto fue: todas las causas de mortalidad o estancia hospitalaria) - 36 sesiones supervisadas ( 3 por semana en banda rodante, caminando o en bicicleta estática durante 12 semanas progresando a 30-35 minutos al 70\% de la frecuencia cardíaca de reserva después de la sexta sesión) seguidas por entrenamiento en casa (40 minutos al 60-70\% de la Frecuencia cardíaca de reserva, 5 veces por semana) vs cuidado usual solo - en 2331 pacientes con IC estable con fracción de eyección $\leq$ $35 \%$ y clase NYHA II-IV seguidos durante un promedio de 30 meses, que encontró que los participantes tenían una edad promedio de 59 años, $28 \%$ eran mujeres y $37 \%$ tenían NYHA III-IV, la etiología del IC fue isquémica en $51 \%$ y la FEVI promedio fue $25 \%$. Un total de 736 pacientes completaron las 36 sesiones supervisadas en
3.9 meses. La adherencia al ejercicio disminuyó de una media de 95 minutos por semana durante el $4^{\circ}$ al $6^{\circ}$ mes de seguimiento a 74 minutos/semana durante el $10^{\circ}$ a $12^{\circ}$ mes y 50 minutos/semana al tercer año. Un poco más del $30 \%$ de los pacientes siempre realizaron más de la meta de tiempo semanal (120 minutos) y al menos el $8 \%$ del grupo control admitió ejercitarse en el período de seguimiento. El EA fue seguro y después de los ajustes de los principales predictores pronósticos del desenlace primario (duración en la prueba de esfuerzo, FEVI, puntaje en "Beck Depresion Inventory II", historia de fibrilación auricular), el EA se asoció con reducciones significativas moderadas de todas las causas de mortalidad u hospitalización (HR 0.89 (95\% CI, 0.81-0.99; $\mathrm{P}=0.03$ ) y de la mortalidad cardiovascular u hospitalizaciones por IC (0.85 (95\% CI, 0.74-0.99; $\mathrm{P}=0.03)^{22}$. También se encontró mejoría significativa moderada en el estado de salud del grupo EA determinado con el "Kansas City Cardiomyopathy Questionnaire" a los 3 meses, que persiste durante el seguimiento, $\mathbf{9 2}$.

\section{Seguridad del Entrenamiento en IC}

Los potenciales riesgos asociados con el ejercicio se deben considerar para garantizar la seguridad del entrenamiento físico en pacientes con IC. Varios factores pueden afectar el riesgo del programa de entrenamiento, siendo los 3 más importantes, la edad, la presencia de enfermedad cardíaca y la intensidad del ejercicio ${ }^{2}$. Los principales riesgos asociados con el ejercicio son la muerte súbita cardíaca y el infarto agudo de miocardio. La incidencia de muerte súbita en pacientes con enfermedad cardíaca es 1 en 60.000 horas-participante siendo más baja en actividades controladas como caminar, bicicleta y banda. Los sujetos menos activos tienen más riesgo de infarto de miocardio durante el ejercicio y actividad física habitual y el fitness cardiovascular tiene una fuerte relación inversa con este riesgo ${ }^{2}$. Meta-análisis, revisiones sistemáticas y el HF-ACTION ilustran la eficacia y seguridad del entrenamiento programado en pacientes con $\mathrm{IC}^{\mathbf{0 - 2 2}, 25}$; un meta-análisis no encontró muertes relacionadas con el ejercicio durante más de 60.000 horas-paciente ${ }^{23}$.

Actualmente, está claro que el ejercicio aeróbico y de fuerza no empeoran la disfunción del ventrículo izquierdo, incluso pueden mejorarla ${ }^{2,7,8,32}$.

Finalmente, una revisión sistemática reciente encontró que los programas de rehabilitación cardíaca que incluyen ejercicio son costo-efectivos para falla cardíaca y enfermedad coronaria ${ }^{49}$; para maximizar los beneficios de cualquier modalidad de entrenamiento, la adherencia a la prescripción del ejercicio para IC u otra enfermedad, se debe evaluar, y determinar, por lo menos, las estrategias para promoverla y las barreras para realizarla. $19,21,50$ 


\section{Referencias:}

1. MCMURRAY JJ,ADAMOPOULOS S, ANKER SD, AURICCHIO A, BOHM M, DICKSTEIN K, et al. ESC Guidelines for the diagnosis and treatment of acute and chronic heart failure 2012: The Task Force for the Diagnosis and Treatment of Acute and Chronic Heart Failure 2012 of the European Society of Cardiology. Developed in collaboration with the Heart Failure Association (HFA) of the ESC. Eur Heart J. England. 2012; 33: 1787-847.

2. MCKELVIE RS. Exercise training in patients with heart failure: clinical outcomes, safety, and indications. Heart Fail Rev. 2008;13:3-11

3. SHIN JJ, HAMAD E, MURTHY S, PINA IL. Heart failure in women. Clin Cardiol. 2012;35:172-7.

4. ROGER VL, GO AS, LLOYD-JONES DM, BENJAMIN EJ, BERRY JD, BORDEN WB, et al. Heart disease and stroke statistics--2012 update: a report from the American Heart Association. Circulation. 2012; 125: e2-e220.

5. TRIPOSKIADIS FK, SKOULARIGIS J. Prevalence and Importance of Comorbidities in Patients With Heart Failure. Curr Heart Fail Rep. 2012;9:354-62.

6. JESSUP M, ABRAHAM WT, CASEY DE, FELDMAN AM, FRANCIS GS, GANIATS TG, et al. 2009 focused update: ACCF/AHA Guidelines for the Diagnosis and Management of Heart Failure in Adults: a report of the American College of Cardiology Foundation/American Heart Association Task Force on Practice Guidelines: developed in collaboration with the International Society for Heart and Lung Transplantation. Circulation. 2009;119:1977-2016.

7. CRIMI E, IGNARRO LJ, CACCIATORE F, NAPOLI C. Mechanisms by which exercise training benefits patients with heart failure. Nat Rev Cardiol. 2009;6:292-300.

8. PAPATHANASIOU G, TSAMIS N, GEORGIADOU P, ADAMOPOULOS S. Beneficial effects of physical training and methodology of exercise prescription in patients with heart failure. Hellenic J Cardiol. 2008;49:267-77.

9. DOWNING J, BALADY GJ. The role of exercise training in heart failure. J Am Coll Cardiol. United States: 2011 American College of Cardiology Foundation. Published by Elsevier Inc; 2011; 58:561-9.

10. COWIE MR. Recent developments in the management of heart failure. Practitioner. 2012;256:25-9, 3 .

11. PIEPOLI MF, GUAZZI M, BORIANI G, CICOIRA M, CORRA U, DALLA LIBERA L, et al. Exercise intolerance in chronic heart failure: mechanisms and therapies. Part I. Eur J Cardiovasc Prev Rehabil. 2010;17:637-42.

12. HAYKOWSKY M, BRUBAKER P, KITZMAN D. Role of physical training in heart failure with preserved ejection fraction. Curr Heart Fail Rep. 2012;9:101-6.

13. PINA IL, APSTEIN CS, BALADY GJ, BELARDINELLI R, CHAITMAN BR, DUSCHA BD, et al. Exercise and heart failure: A statement from the American Heart Association Committee on exercise, rehabilitation, and prevention. Circulation. 2003;107:1210-25.

14. DUSCHA BD, SCHULZE PC, ROBBINS JL, FORMAN DE Implications of chronic heart failure on peripheral vasculature and skeletal muscle before and after exercise training. Heart Fail Rev. 2008;13:21-37.

15. CAHALIN LP, CHASE P, ARENA R, MYERS J, BENSIMHON D, PEBERDY MA, et al. A meta-analysis of the prognostic significance of cardiopulmonary exercise testing in patients with heart failure. Heart Fail Rev. 2013;18:79-94.

16. GEORGIADOU P, ADAMOPOULOS S. Skeletal muscle abnormalities in chronic heart failure. Curr Heart Fail Rep. 2012;9:128-32.

17. BRAITH RW, BECK DT. Resistance exercise: training adaptations and developing a safe exercise prescription. Heart Fail Rev. 2008;13:69-79.

18. YUNG LM, LAHER I, YAO X, CHEN ZY, HUANG Y, LEUNG FP. Exercise, vascular wall and cardiovascular diseases: an update (part 2). Sports Med. 2009;39:45-63.

19. PIEPOLI MF, CONRAADS V, CORRA U, DICKSTEIN K, FRANCIS DP, JAARSMA T, et al. Exercise training in heart failure: from theory to practice. A consensus document of the Heart Failure Association and the European Association for Cardiovascular Prevention and Rehabilitation. Eur J Heart Fail. 2011;13:347-57

20. TAYLOR RS, DAVIES EJ, DALAL HM, DAVIS R, DOHERTY P, COOPER C, et al. Effects of exercise training for heart failure with preserved ejection fraction: A systematic review and meta-analysis of comparative studies. Int J Cardiol. 2012;162:6-13

21. DAVIES EJ, MOXHAM T, REES K, SINGH S, COATS AJ, EBRAHIM S, et al. Exercise training for systolic heart failure: Cochrane systematic review and meta-analysis. Eur J Heart Fail. 2010;12:706-15.

22. O'CONNOR CM, WHELLAN DJ, LEE KL, KETEYIAN SJ, COOPER LS, ELLIS SJ, et al. Efficacy and safety of exercise training in patients with chronic heart failure: HF-ACTION randomized controlled trial. JAMA. 2009;301:1439-50.

23. SMART N, MARWICK TH. Exercise training for patients with heart failure: a systematic review of factors that improve mortality and morbidity. Am J Med. 2004:693-706.

24. PIEPOLI MF, DAVOS C, FRANCIS DP, COATS AJ. Exercise training meta-analysis of trials in patients with chronic heart fai- 
lure (ExTraMATCH). BMJ. 2004;328:189.

25. VAN DER MEER S, ZWERINK M, VAN BRUSSEL M, VAN DER VALK P, WAJON E, VAN DER PALEN J. Effect of outpatient exercise training programmes in patients with chronic heart failure: a systematic review. Eur J Cardiovasc Prev Rehabil; 2011.

26. MYERS J. Principles of exercise prescription for patients with chronic heart failure. Heart Fail Rev. 2008;13:61-8.

27. MEZZANI A, CORRA U, GIANNUZZI P. Central adaptations to exercise training in patients with chronic heart failure. Heart Fail Rev. 2008;13:13-20.

28. BEER M, WAGNER D, MYERS J, SANDSTEDE J, KÖSTLER H, HAHN D, NEUBAUER S, DUBACH P. Effects of exercise training on myocardial energy metabolism and ventricular function assessed by quantitative phosphorus-31 magnetic resonance spectroscopy and magnetic resonance imaging in dilated cardiomyopathy. J Am Coll Cardiol 2008;51:1883-91.

29. MALFATTO G, BRANZI G, OSCULATI G, VALLI P, CUOCCIO P, CIAMBELLOTTI F, et al. Improvement in left ventricular diastolic stiffness induced by physical training in patients with dilated cardiomyopathy. J Card Fail. 2009;15:327-33.

30. KETEYIAN SJ. Exercise training in congestive heart failure: risks and benefits. Prog Cardiovasc Dis. United States: 2011 Elsevier Inc; 2011;53:419-28.

31. HAYKOWSKY MJ, LIANG Y, PECHTER D, JONES LW, MCALISTER FA, CLARK AM. A meta-analysis of the effect of exercise training on left ventricular remodeling in heart failure patients: the benefit depends on the type of training performed. $\mathrm{J}$ Am Coll Cardiol. 2007;49:2329-36.

32. CHEN YM, LI ZB, ZHU M, CAO YM. Effects of exercise training on left ventricular remodelling in heart failure patients: an updated meta-analysis of randomised controlled trials. Int J Clin Pract. 2012;66:782-91.

33. KEMI OJ, HOYDAL MA, HARAM PM, GARNIER A, FORTIN D, VENTURA-CLAPIER R, et al. Exercise training restores aerobic capacity and energy transfer systems in heart failure treated with losartan. Cardiovasc Res. 2007;76:91-9.

34. PERSEGHIN G, DE COBELLI F, ESPOSITO A, BELLONI E, LATTUADA G, CANU T, et al. Left ventricular function and energy metabolism in middle-aged men undergoing long-lasting sustained aerobic oxidative training. Heart. 2009;95:630-5.

35. VENTURA-CLAPIER R. Exercise training, energy metabolism, and heart failure. Appl Physiol Nutr Metab. 2009;34:336-9.

36. SMART NA, MEYER T, BUTTERFIELD JA, FADDY SC, PASSINO C, MALFATTO G, et al. Individual patient metaanalysis of exercise training effects on systemic brain natriuretic peptide expression in heart failure. Eur J Prev Cardiol. 2012;19:428-35.

37. NIEBAUER J. Effects of exercise training on inflammatory markers in patients with heart failure. Heart Fail Rev. 2008;13:39-49.
38. BRUM PC, BACURAU AV, MEDEIROS A, FERREIRA JC, VANZELLI AS, NEGRAO CE. Aerobic exercise training in heart failure: impact on sympathetic hyperactivity and cardiac and skeletal muscle function. Braz J Med Biol Res. 2011;44:827-35.

39. PATEL KP, ZHENG H. Central neural control of sympathetic nerve activity in heart failure following exercise training. Am J Physiol Heart Circ Physiol. 2012;302:H527-37.

40. NEGRAO CE, MIDDLEKAUFF HR. Adaptations in autonomic function during exercise training in heart failure. Heart Fail Rev. 2008;13:51-60.

41. SMART NA, STEELE M. The effect of physical training on systemic proinflammatory cytokine expression in heart failure patients: a systematic review. Congest Heart Fail. 2011;17:110-4.

42. GIELEN S, ADAMS V, MOBIUS-WINKLER S, LINKE A, ERBS S, YU J, et al. Anti-inflammatory effects of exercise training in the skeletal muscle of patients with chronic heart failure. $\mathrm{J}$ Am Coll Cardiol. 2003;42:861-8.

43. HAMBRECHT R, SCHULZE PC, GIELEN S, LINKE A, MOBIUS-WINKLER S, ERBS S, et al. Effects of exercise training on insulin-like growth factor-I expression in the skeletal muscle of non-cachectic patients with chronic heart failure. Eur J Cardiovasc Prev Rehabil. 2005;12:401-6.

44. BJORNSTAD HH, BRUVIK J, BJORNSTAD AB, HJELLESTAD BL, DAMAS JK, AUKRUST P. Exercise training decreases plasma levels of soluble CD40 ligand and P-selectin in patients with chronic heart failure. Eur J Cardiovasc Prev Rehabil. 2008;15:43-8.

45. GADEMAN MG, SWENNE CA, VERWEY HF, VAN DE VOOREN H, HAEST JC, VAN EXEL HJ, et al. Exercise training increases oxygen uptake efficiency slope in chronic heart failure. Eur J Cardiovasc Prev Rehabil. 2008;15:140-4.

46. SMART NA, DIEBERG G, GIALLAURIA F. Intermittent versus continuous exercise training in chronic heart failure: A metaanalysis. Int J Cardiol: 2011 Elsevier Ireland Ltd; 2011.

47. SMART NA, GIALLAURIA F, DIEBERG G. Efficacy of inspiratory muscle training in chronic heart failure patients: A systematic review and meta-analysis. Int J Cardiol: Crown 2012. Published by Elsevier Ireland Ltd; 2012.

48. BLUMENTHAL JA, BABYAK MA, O'CONNOR C, KETEYIAN S, LANDZBERG J, HOWLETT J, et al. Effects of exercise training on depressive symptoms in patients with chronic heart failure: the HF-ACTION randomized trial. JAMA. 2012:465-74.

49. WONG WP, FENG J, PWEE KH,LIM J.A systematic review of economic evaluations of cardiac rehabilitation. BMC Health Serv Res; 2012;12:243.

50. TIERNEY S, MAMAS M, WOODS S, RUTTER MK, GIBSON M, NEYSES L, et al. What strategies are effective for exercise adherence in heart failure? A systematic review of controlled studies. Heart Fail Rev. 2012;17:107-15. 